\title{
Selected Topics on Advanced Information Systems Engineering: Editorial Introduction to the Issue 5 of CSIMQ
}

\author{
Janis Grabis ${ }^{1}$, Kurt Sandkuhl ${ }^{2}$ \\ ${ }^{1}$ Riga Technical University, Institute of Information Technology, \\ Kalku 1, LV-1658, Riga, Latvia \\ ${ }^{2}$ University of Rostock, Chair Business Information Systems, \\ Albert-Einstein-Str. 22, 18059 Rostock, Germany \\ Grabis@rtu.lv, Kurt.Sandkuhl@uni-rostock.de
}

Information systems engineering draws its foundation from various interrelated disciplines including, e.g., conceptual modeling, database systems, business process management, requirements engineering, human computer interaction, and enterprise computing to address various practical challenges in development and application of information systems. During the last years, such aspects as Creativity, Ability, and Integrity have attained a major importance in Information Systems Engineering [1]. Creativity implies designing Information Systems in novel and unexpected ways making use of information from different sources that need to be merged and molded to become meaningful and valuable. Ability enables systems to be capable to deliver business in excellent, competitive, and agile ways. Integrity refers to ensuring security, trustworthiness, and compliance with ethical code of information systems. Creativity, Ability, and Integrity were the guiding subjects of the 27th edition of CAISE, the international Conference on Advanced Information Systems engineering, which took place in June 2015 in Stockholm, Sweden.

This issue of the journal on Complex Systems Informatics and Modeling (CSIMQ) presents selected papers from the CAiSE Forum 2015, which was part of the CAiSE 2015 conference. The CAiSE Forum aimed at presenting and discussing new ideas and tools related to information systems engineering. All selected papers were substantially extended and include new material and new research results as compared to the previous versions published in [2]. Based on a thorough review process according to the CSIMQ standards, five papers were accepted for Issue 5 of CSIMQ. These papers address different technical and methodic aspects of information systems engineering:

- Merethe Heggset, John Krogstie, and Harald Wesenberg base their paper "The Influence of Syntactic Quality on Pragmatic Quality of Enterprise Process Models" on statistics and experiences collected in the Norwegian oil company Statoil. Guided by the SEQUAL quality framework, the authors identify challenges regarding pragmatic quality of enterprise process models and propose measures how to achieve higher quality. One of the main challenges identified for a number of models was that they had poorer syntactic quality than other models of the same size. Improving the syntactic quality gave mixed results.

- Antoni Olivé investigates a problem of selecting appropriate Commercial-off-the-shelf (COTS) systems in the paper "A Formal Method for Conceptual Fit Analysis". The article approaches the COTS selection problem from the perspective of evaluation of conceptual fit. A method, which formally determines the conceptual misfits between the user requirements and a set of candidate systems, is elaborated. The important feature of the 
method is possibility to integrate it with existing COTS selection methods. The conceptual fit analysis provides means for formal evaluation of such criteria as Conceptual deficits and incompatibilities and Conceptual excess.

- Marco Alessi, Alessio Camillò, Valentina Chetta, Enza Giangreco, Mona Soufivand, and Davide Storelli present a paper on "Applying Idea Management System (IMS) Approach to Design and Implement a collaborative Environment in Public Service related open Innovation Processes". The authors propose an idea management framework consisting of a life-cycle and actions supporting each life-cycle phase. The proposed framework was tested in the municipality of Lecce (Italy). Lecce used direct participation of the citizens in the context of the candidacy as European Capital of Culture. The use of the framework and its supporting tools was perceived as beneficial for stimulating value co-creation between citizens and the municipal government.

- Sagar Sunkle, Deepali Kholkar, and Vinay Kulkarni position their contribution in the field of governance, risk, and compliance. They propose the use of semantic matching in order to identify correspondences and mappings between terms used in regulations and business process models or other operational specifics. More concrete, they use Semantics of Business Vocabulary Rules (SBVR) to model vocabularies of regulations and operational concepts as elaborated text. The paper has the title "Toward Better Mapping between Regulations and Operations of Enterprises Using Vocabularies and Semantic Similarity".

- Jorge Echeverria, Jaime Font, Oscar Pastor, and Carlos Cetina focus on the general problem of providing an adequate tool support in information systems engineering as a prerequisite for successful adoption of engineering methods. Specifically, their paper "Usability Evaluation of Variability Modeling by means of Common Variability Language" evaluates usability of Common Variability Language and a corresponding tool. The evaluation is performed by means experimental studies. The usability problems and potential solutions for these problems are identified as the results of these studies.

The CSIMQ editorial team would like to thank external reviewers of this issue Gundars Alksnis, Erika Asnina, Giuseppe Berio, Solvita Berzisa, Michael Fellmann, Dimitris Karagiannis, Marite Kirikova, Regine Laleau, Birger Lantow, Michael Leyer, Sotirios Liaskos, Gilles Perrouin, Geert Poels, Christoph Quix, Jolita Ralyté, Guttorm Sindre, Inese Supulniece, and Gianluigi Viscusi, as well as the regular members of the International Editorial Review Board of the journal, for providing valuable comments and improvement proposals for the papers.

\section{References}

[1] J. Zdravkovic, M. Kirikova, P. Johannesson, Advanced Information Systems Engineering, 27th International Conference, CAiSE 2015, Stockholm, Sweden, June 8-12, 2015, Proceedings, Lecture Notes in Computer Science 9097, Springer, 2015. Available: http://dx.doi.org/10.1007/978-3-319-19069-3

[2] J. Grabis, K. Sandkuhl, Proceedings of the CAiSE 2015 Forum at the 27th International Conference on Advanced Information Systems Engineering co-located with 27th International Conference on Advanced Information Systems Engineering, CAiSE 2015, Stockholm, Sweden, June 8-12, 2015, CEUR Workshop Proceedings, Vol. 1367. Aachen: RWTH, 2015. [Online]. Available: http://ceur-ws.org/Vol-1367/ 